\title{
A radiological method of following changes and displacements of the brain after surgery ${ }^{1}$
}

\author{
S. OBRADOR AND V. QUEIMADELOS \\ From the Departments of Neurosurgery of the Instituto de Investigaciones Medicas \\ and the Gran Hospital de la Beneficiencia General, Madrid
}

Modern radical neurosurgical techniques and the advances in the post-operative care of the patients, such as nasal feeding, tracheotomies, the giving of corticosteroids, and the use of urea and other substances for the treatment of cerebral oedema, have greatly improved the outlook of patients submitted to intracranial operations. There are still a number of cases with a bad post-operative course, especially those with large infiltrating tumours of the brain where only palliative operations can be performed.

In order to follow the local and general changes in the brain after an operation for a tumour or other expanding lesion of the cerebral hemispheres we have developed a simple radiological method which is described here.

\section{MATERIAL AND METHODS}

Fifty consecutive patients operated on for tumour or other expanding intracranial lesions during the last few months were studied.

The predominant localization of the intracranial lesions was as follows:

Frontal lobes.........................14

Temporal lobes.........................16

Parietal lobes............................17

Occipital lobes ....................... 3

The conditions for which the operations were performed are the following:

Glioma (glioblastoma multiforme 15) ..........31

Meningioma ................................. 10

Metastatic carcinoma ................... 3

Abscess ............................ 3

Hydatid cyst $\ldots \ldots \ldots \ldots \ldots \ldots \ldots \ldots \ldots \ldots \ldots \ldots, 2$

Intracerebral haematoma $\ldots \ldots \ldots \ldots \ldots \ldots \ldots 1$

The neurosurgical operations performed in these 50 patients were:

Total removal of the lesion.................23

Lobectomy in gliomas...................13

Partial removal..................................

Biopsy and decompression................. 2

${ }^{1}$ Paper presented at the Second European Congress of Neuosurgery, Rome, April 1963.
During the operations no silver clips were used (incidentally we rarely use clips for operations on tumours). Once the removal of the lesion was completed, however, we placed several silver clips at different points on the surface and in the depths of the surgical cavity (between two to eight clips but usually four to six) in order to facilitate radiological study.

Immediately after the operation the first radiograph (control) was taken in perpendicular antero-posterior and lateral projections of the skull. The technical conditions were kept constant for the studies which followed. The next radiograph was taken 24 hours afterwards, and daily studies were then made in the first group of patients. In the remainder of the patients the radiographic studies were carried out 24 hours after operation and then only every two to four days, provided that the clinical progress was satisfactory. Such studies were continued for several weeks or months after the operation. In a few patients the radiographs were taken hourly or every few hours during a study of the action of intravenous urea.

The radiological material was studied and analysed in two ways; first by plotting the distances between certain of the clips and then the distances to various cranial lines. In the antero-posterior projection we took a midline (A) perpendicular to the centre of a horizontal line which passes through the intersection of the large sphenoidal wings with the orbits. Another midline (B) was traced as a perpendicular to the centre of a horizontal line joining the internal edge of the orbital shadows. The separation of these two lines should not be more than $3 \mathrm{~mm}$. In the lateral view of the skull the situation of the clips was related to lines drawn by the prolongation of the clivus and its perpendicular passing by the base of the anterior clinoids.

These measurements are liable to error owing to radiographic distortions, and the correct radiographic projections may be rendered difficult because of lack of cooperation by the patient. The oedema of the scalp and face, the bandages, etc., may also make it difficult to obtain the proper position for the radiological study. Other circumstances, such as the results of decompression in some patients, low temporal flaps, or marked flexion of the head with the projection of the petrous bones in the orbits, may interfere with tracing the cranial lines of reference.

The antero-posterior projection is, in our experience, the most useful for our purpose but it is extremely important to select those deep and superficial clips situated 


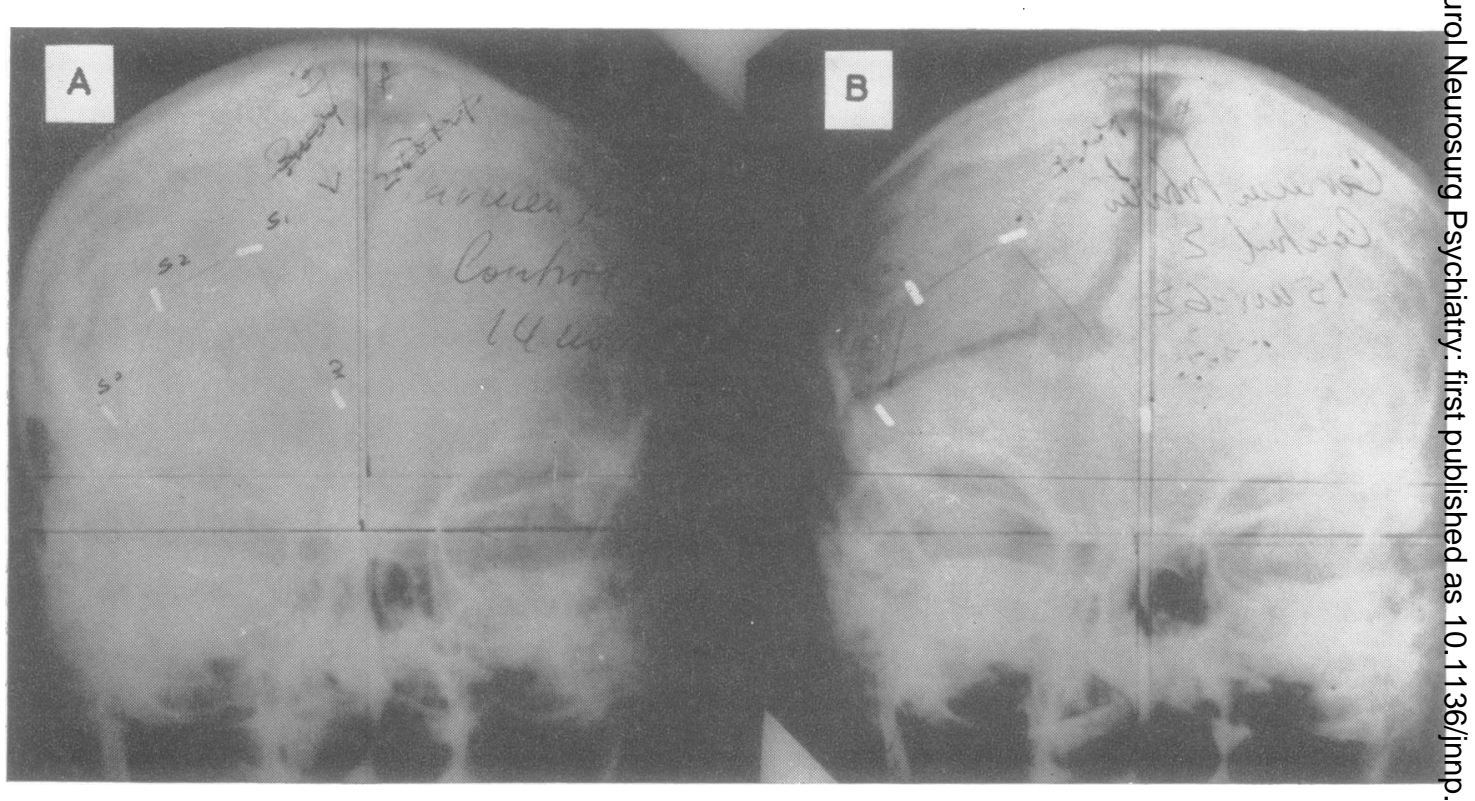

FIG. 1. Frontal astrocytoma. (a) Control radiological study immediately after operation of partial removal; (b) one day. N afterwards showing the increase of the inter-clip distances due to cerebral oedema.

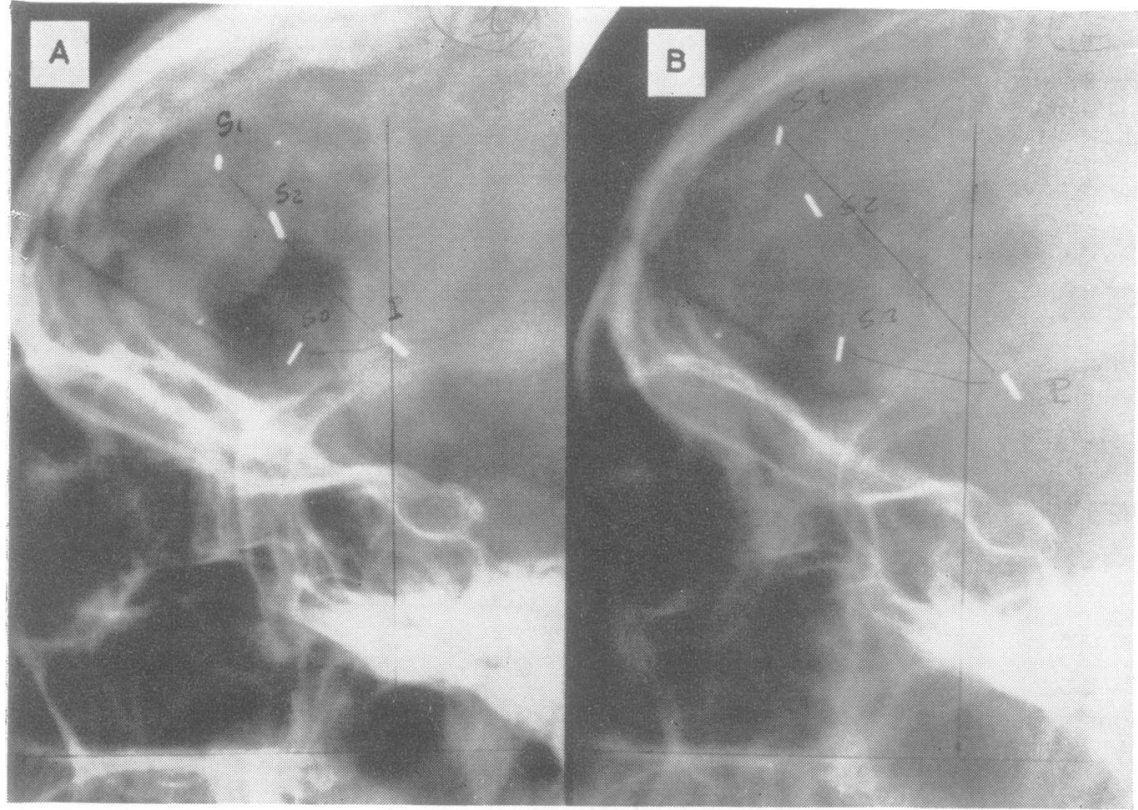

FIG. 2. The lateral views of the same case as in Figure 1. (a) Control after the operation; (b) 72 hours later. 


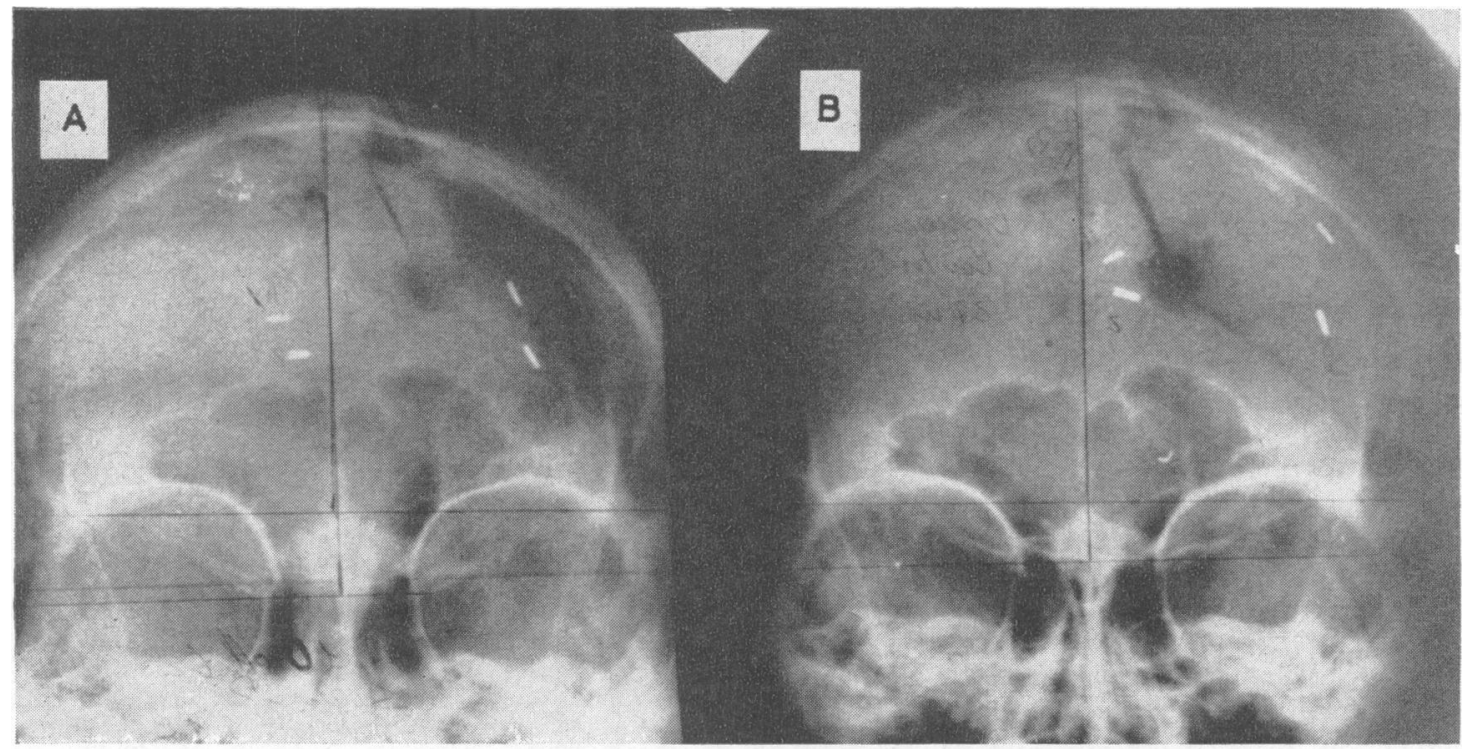

FIG. 3. Frontal glioma. (a) Collapse of the brain as shown by the position of the clips with generalized displacement towards the midline immediately after the operation; (b) 17 days later.

in or near to the radiological planes we use. In this way we can largely eliminate the possibility of mistakes due to distortions. For this reason it is important to place several clips in the operative cavity in order to obtain the type of measurement we require.

As examples of these changes in Fig. 1a is shown the film immediately after the partial removal of a frontal astrocytoma and in Fig. 1b the changes which appeared 24 hours afterwards. Figure 2 is the lateral view of the same case after operation (Fig. 2a) and 72 hours afterwards (Fig. 2b). In Fig. 3a we have evidence of brain shrinkage immediately after the operation for a frontal glioma and the situation of the clips in the later postoperative course 17 days later (Fig. 3b).

The other method we have used to determine the position and modifications of the clips is to superimpose the different films on each other.

In general the first method of measuring the different inter-clip distances and of the distances to the midline is more precise although still liable to error, but the second method is simple and effective when there are marked changes.

\section{RESULTS}

THE POST-OPERATIVE PERIOD The first fact demonstrated by our method is that the local and general changes which occur in the brain after the total or partial removal of a space-occupying lesion appear slowly and last a considerable time. The most frequent finding (caused by a varying degree of cerebral oedema) is an increase of the inter-clip distance and modifications of the distances to the midline (Fig. 1). We have also seen post-operative states of collapse or diminution of the brain volume characterized by displacement of both the superficial and the deep clips towards the midline without important modifications of the inter-clip distances

TABLE I

SUMMARY OF RESULTS

Radiological Findings

Cases with Good

Post-operative Course

Cases with Bad Post-operative Course

Minimal changes of the clip distances (less than $5 \mathrm{~mm}$.)

Initial diminution of brain volume

Brain shrinkage followed by moderate cerebral oedema during the first 3 weeks

Cerebral swelling in the first 3 weeks

Shrinkage followed by persistent cerebral oedema beyond 3 weeks

Persistent cerebral swelling beyond 3 weeks

10
2

6

14
3

4

Total 50 
(Fig. 3). Finally, in a number of patients there are only minimal variations in the position of the clips.

We can separate our 50 patients into two groups. In the first are 39 patients who made a good postoperative recovery. In the second are 11 patients with a bad post-operative course associated with severe impairment of consciousness requiring nasal feeding and tracheotomy. Table I summarizes the radiological findings in the two groups.

According to our radiological method, about half of the patients (20) with a good post-operative course after the surgical treatment of a tumour or other space-occupying lesion of the cerebral hemispheres developed a moderate degree of cerebral oedema or swelling during the first three weeks after the operation (Fig. 4). Ten patients only showed minimal radiological changes and two initial cerebral shrinkage with diminution of brain volume. In only seven cases of this favourable group did cerebral swelling or oedema persist beyond the first three weeks. In contrast, most of the cases (nine out of 11) with the bad post-operative course showed persistent cerebral oedema or brain swelling which tended to be progressive and of irregular character for several weeks after the operation (Fig. 5).

ACTION OF THE UREA AND OTHER OBSERVATIONS The effect of the administration of intravenous urea in causing a diminution of the brain volume has been mentioned, and of the 17 patients of the total group who received urea at the operation 15 presented during the first 24 hours signs of cerebral shrinkage with a general displacement of the superficial and deep clips towards the midline but without great changes in the inter-clip distances. As we have also seen (Table I), brain shrinkage was followed in most of the patients (13) by varying degrees of cerebral oedema.

We also tested the action of intravenous urea in three patients during the post-operative course with frequent radiological controls every hour or every few hours. We found in these conditions quick changes of the superficial and deep clips with midline displacements lasting from four to six hours. This action of urea seems to be more marked in the immediate post-operative period when there is a tendency for oedema to develop.

Occasionally we have seen striking changes in position of the clips due to their being dislodged. The displacement of the clips may also indicate the development of post-operative collections of fluid, blood, or pus. In this connexion it may be mentioned that the late Dr. Van Wagenen used to apply a silver clip to the surface of the dura as an indicator for recognizing epidural haematomas.

In two patients with parasagittal meningiomas
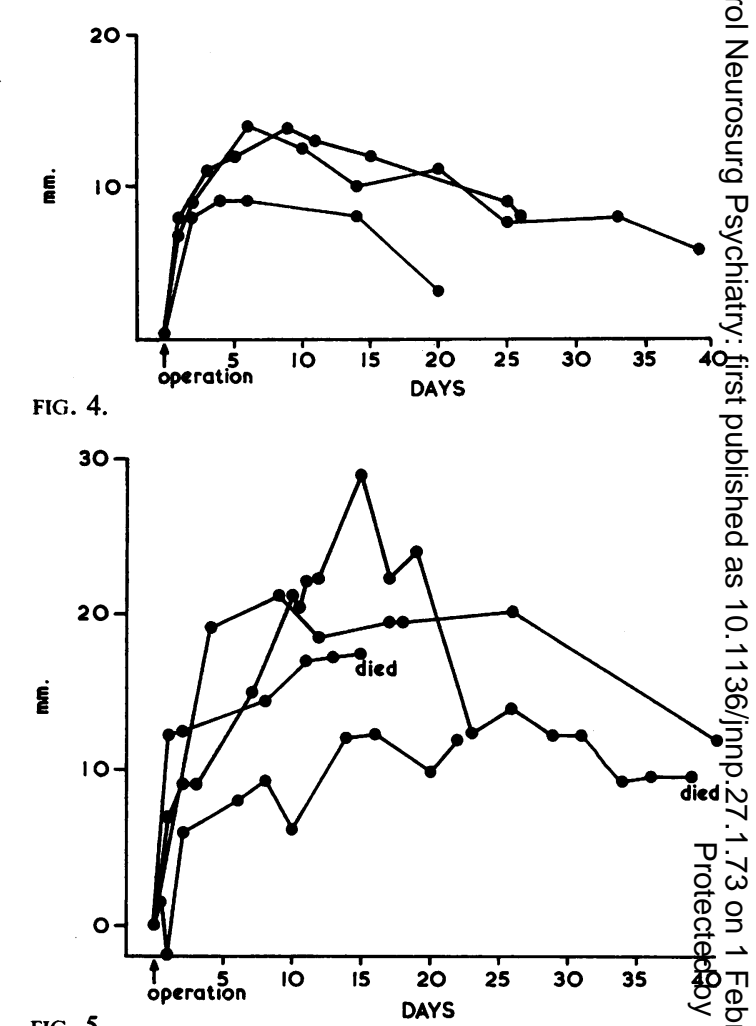

FIG. 5.

FIGS. 4 and 5. Comparison of the degree of cerebsat oedema measured after operation in patients with a gō d post-operative course (Fig. 4) and in those with a bad post-operative course (Fig. 5) related to the number of days after operation in which it was observed.

clips were placed on the falx for midline references

In several cases there has been no definite corre lation between the intracranial pressure as measured by lumbar puncture and the modifications in the position of the clips.

Finally, in five patients with gliomas submitted tor post-operative radiation treatment, we have not seen much change in the radiological studies.

Clinical APPLications of THE METHOD Since the completion of this study in the first series of 50 cases 3 . we have adopted it in our Service as a routine proin cedure in certain patients, especially in those with infiltrating gliomas. We take radiographs in the usual antero-posterior and lateral projections immediately after the operation, but in the post $\frac{7}{0}$ operative period we now only repeat these radio logical studies when indicated by the clinical con $N$ dition of the patient. By this means we can recognizo

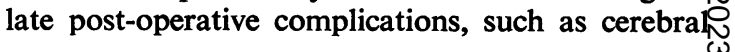


shrinkage, that require an entirely different therapeutic approach from those associated with cerebral swelling. The presence of haematomas or other complicating collections of fluid or secondary to late infections can also be detected.

In the late stages of the follow-up this method can also be useful in outlining the site for radiation therapy.

\section{SUMMARY}

In 50 patients operated on for tumours and other space-occupying lesions of the cerebral hemispheres several silver clips were placed in the surgical cavities and their subsequent changes in position were followed radiologically.

Cerebral oedema was the most frequent postoperative finding and produced in the radiological study an increase of certain inter-clip distances. Shrinkage or diminution of brain volume could also be recognized.

The radiological findings were studied in relation to the patients' clinical progress and the various factors involved were analysed.

Intravenous urea was shown to induce a state of brain shrinkage lasting several hours and this condition was also seen in patients given urea immediately after operation. 